\title{
VISCOPLASTICITY FOR INSTABILITIES DUE TO STRAIN SOFTENING AND STRAIN-RATE SOFTENING*
}

\author{
W. M. WANG, L. J. SLUYS** AND R. DE BORST ${ }^{\dagger}$ \\ Delft University of Technology, Faculty of Civil Engineering, P.O. Box 5048, 2600 GA Delft, The Netherlands
}

\begin{abstract}
Three viscoplastic approaches are examined in this paper. First, the overstress viscoplastic models (i.e. the Perzyna model and the Duvaut-Lions model) are outlined. Next, a consistency viscoplastic approach is presented. In the consistency model a rate-dependent yield surface is employed while the standard Kuhn-Tucker conditions for loading and unloading remain valid. For this reason, the yield surface can expand and shrink not only by softening or hardening effects, but also by softening/hardening rate effects. A full algorithmic treatment is presented for each of the three models including the derivation of a consistent tangential stiffness matrix. Based on a limited numerical experience it seems that the consistency model shows a faster global convergence than the overstress approaches. For softening problems all three approaches have a regularising effect in the sense that the initial-value problem remains well-posed. The width of the shear band is determined by the material parameters and, if present, by the size of an imperfection. A relation between the length scales of the three models is given. Furthermore, it is shown that the consistency model can properly simulate the so-called S-type instabilities, which are associated with the occurrence of travelling Portevin-Le Chatelier bands. (C) 1997 John Wiley \& Sons, Ltd.
\end{abstract}

Int. J. Numer. Meth. Engng., 40, 3839-3864 (1997)

No. of Figures: $13 . \quad$ No. of Tables: $0 . \quad$ No. of References: 16.

KEY WORDS: viscoplasticity; instability; strain softening; strain-rate softening; tangential stiffness matrix

\section{INTRODUCTION}

The calculation of shear bands and other material instability phenomena has become of considerable interest because of its importance in predicting failure of materials. The numerical simulation of shear bands with classical rate-independent softening models fails because, under dynamic loading conditions, the field equations that describe the motion of the body may lose hyperbolicity when strain softening occurs. The initial value problem becomes ill-posed and numerical solutions suffer from mesh sensitivity. Because the wave speed in the softening region becomes imaginary, loading waves are not able to propagate and the localization zone stays confined to a line with zero thickness. ${ }^{1}$ These results are in contradiction with experimental data, which show finite widths of the localization zone.

In order to solve the mesh-sensitivity problem, viscous terms can be introduced to keep the field equations hyperbolic. Needleman, ${ }^{2}$ Loret and Prevost ${ }^{3}$ and Sluys ${ }^{4}$ have shown that material

* Dedicated to Prof. Dr. -Ing. E. Stein on the occasion of his 65 th birthday

** Correspondence to: L. J. Sluys, Faculty of Civil Engineering, Delft University of Technology, P.O. Box 5048, NL-2600

GA Delft, The Netherlands

${ }^{\dagger}$ Also at Eindhoven University of Technology, Faculty of Mechanical Engineering

CCC 0029-5981/97/203839-26\$17.50

Received 16 January 1996

(C) 1997 John Wiley \& Sons, Ltd.

Revised 23 July 1996 
rate dependence introduces a length scale effect into the initial value problem under dynamic loading conditions even though the constitutive equations do not explicitly contain a parameter with the dimension of length. Sluys ${ }^{4}$ has demonstrated that this viscous length scale effect can be related to the spatial attenuation of waves that have real wave speeds in the softening regime. Wang et al. ${ }^{5}$ related the internal length scale to the softening modulus and examined the influence of an imperfection (see also Reference 6). They showed that the width of the localization zone is determined by the smallest value of the internal length scale and the size of the imperfections.

In this paper a review of viscoplastic models and their algorithmic aspects will be given. First, the overstress viscoplastic methods according to Perzyna ${ }^{7-9}$ and Duvaut-Lions ${ }^{10,11,3}$ will be investigated. These viscoplastic models are implemented by allowing the stress state to be outside the yield surface and directly define the plastic relaxation equations in the stress space. As an alternative model, the consistency approach is presented. Now the viscoplastic regularization is achieved by introducing a rate-dependent yield surface. The standard Kuhn-Tucker conditions for rate-independent plasticity then still apply. For this reason, the yield function can expand and shrink not only by hardening or softening effects, but also by hardening rate or softening rate effects. This has the advantage that the model can simulate both H-type instabilities (i.e. stationary shear bands) and S-type instabilities (i.e. propagative Portevin-Le Chatelier bands ${ }^{12}$ ).

In this paper we will first discuss the algorithmic aspects of the three viscoplastic approaches. Next, it is shown that the three models have a regularizing effect in the sense that they keep the field equations hyperbolic under dynamic loading conditions. Accordingly, the initial-value problem remains well-posed. By a wave dispersion analysis it is demonstrated that rate-dependent models naturally introduce a length scale parameter into the initial value problem. A relation between the length scales of the three models is given. Finally, numerical examples are presented of $\mathrm{H}$ - and S-type instabilities and some evidence is given that the consistency model exhibits a faster convergence than the algorithms for the Perzyna and the Duvaut-Lions models.

\section{RATE-INDEPENDENT PLASTICITY}

We consider a body $B$ with volume $\Omega$ and surface $\partial \Omega=\partial_{\mathbf{t}} \Omega \cup \partial_{\mathbf{u}} \Omega$, where $\partial_{\mathbf{t}} \Omega$ and $\partial_{\mathbf{u}} \Omega$ are the traction and kinematic boundaries, respectively, with $\partial_{\mathbf{t}} \Omega \cap \partial_{\mathbf{u}} \Omega=0$. The equations of motion, the kinematic equations for small displacement gradients and the corresponding initial and boundary conditions read

$$
\begin{array}{ll}
\nabla \cdot \boldsymbol{\sigma}+\mathbf{p}=\rho \ddot{\mathbf{u}} & \text { in } \Omega \\
\dot{\boldsymbol{\varepsilon}}=\frac{1}{2}\left(\boldsymbol{\nabla} \mathbf{v}+\nabla \mathbf{v}^{\mathrm{T}}\right) & \text { in } \Omega \\
\mathbf{u}(x, 0)=\mathbf{u}_{\mathbf{0}}, \quad \mathbf{v}(x, 0)=\boldsymbol{v}_{0} & \text { in } \Omega \\
\mathbf{t}=\overline{\mathbf{n}} \cdot \boldsymbol{\sigma} & \text { on } \partial_{\mathbf{t}} \Omega \\
\mathbf{u}(x, t)=\overline{\mathbf{u}} & \text { on } \partial_{\mathbf{u}} \Omega
\end{array}
$$

in which $\boldsymbol{\varepsilon}$ and $\boldsymbol{\sigma}$ denote the strain and stress tensors, $\rho$ is the density, $\mathbf{u}, \mathbf{v}, \mathbf{p}$ and $\mathbf{t}$ represent the displacement, velocity, body force and surface traction with the outward normal $\overline{\mathbf{n}}$, the symbol $\mathbf{\nabla}$, $(\cdot)$ and ()$^{T}$ are the gradient operator, the inner tensor product and the transpose symbol, and a superimposed dot denotes differentiation with respect to time, respectively. The constitutive 
equations for rate-independent plasticity can be written in a rate format as

$$
\begin{aligned}
\dot{\boldsymbol{\varepsilon}} & =\boldsymbol{\varepsilon}^{\mathrm{e}}+\dot{\boldsymbol{\varepsilon}}^{\mathrm{p}} \\
\dot{\boldsymbol{\sigma}} & =\mathbf{D}^{\mathrm{e}}:\left(\dot{\boldsymbol{\varepsilon}}-\dot{\boldsymbol{\varepsilon}}^{\mathrm{p}}\right) \\
\dot{\boldsymbol{\varepsilon}}^{\mathrm{p}} & =\dot{\lambda} \mathbf{n}
\end{aligned}
$$

where $\mathbf{D}^{\mathrm{e}}$ is the elasticity tensor, the symbol ( : ) represents the doubly contracted tensor product, $\mathbf{n}$ is the direction of the plastic flow and $\dot{\lambda}$ is the plastic multiplier. The yield criterion of inviscid plasticity and the loading-unloading conditions can be expressed in Kuhn-Tucker form as

$$
\dot{\lambda} \geqslant 0, \quad f \leqslant 0, \quad \dot{\lambda} f=0
$$

where $f(\boldsymbol{\sigma}, \kappa)$ is the yield function and $\kappa$ is the equivalent plastic strain which describes the plastic deformation history. In this paper a strain-hardening/softening hypothesis is assumed

$$
\dot{\kappa}=\sqrt{2 / 3 \dot{\boldsymbol{\varepsilon}}^{\mathrm{p}}: \dot{\boldsymbol{\varepsilon}}^{\mathrm{p}}}
$$

For algorithmic convenience we limit our interest to Von Mises plasticity with an associated flow rule

$$
f(\boldsymbol{\sigma}, \kappa)=\sqrt{3 J_{2}}-\bar{\sigma}(\kappa), \quad \mathbf{n}=\frac{\partial f}{\partial \boldsymbol{\sigma}}
$$

in which $J_{2}$ is the second invariant of the deviatoric stress tensor and $\bar{\sigma}$ is a hardening-softening function with a modulus $h=\partial \bar{\sigma} / \partial \kappa$. Substitution of equations (8) and (11) into equation (10) gives

$$
\dot{\kappa}=\dot{\lambda}
$$

so that the yield function can also be expressed as $f(\boldsymbol{\sigma}, \lambda)$.

In the context of finite element analysis, the equation of motion (1) is normally fulfilled in a weak form, i.e.

$$
\int_{\Omega}[\boldsymbol{\sigma}: \delta \boldsymbol{\varepsilon}+\rho \ddot{\mathbf{u}} \cdot \delta \mathbf{u}] \mathrm{d} \Omega=\int_{\partial_{t} \Omega} \mathbf{t} \cdot \delta \mathbf{u} \mathrm{d} S
$$

where the virtual displacement $\delta \mathbf{u}$ is zero on the kinematic boundary $\partial_{\mathbf{u}} \Omega$ and the body force $\mathbf{p}$ is neglected. To arrive at a semi-discretized set of equations equation (13) is solved by means of finite elements. Next, the second-order ordinary differentiation equations are discretized in time, e.g. through the Newmark method, which results a set of fully discrete algebraic equations. ${ }^{13}$

\section{RATE-DEPENDENT PLASTICITY}

In this section three viscoplastic models are presented: the Perzyna and Duvaut-Lions viscoplastic models and the consistency model. In the overstress viscoplastic approaches the plastic flow is constrained by the plastic relaxation equation. In the consistency viscoplastic model the viscoplastic flow is determined by the Kuhn-Tucker conditions with a rate-dependent yield function. 


\subsection{Perzyna viscoplastic model}

In the viscoplastic model according to Perzyna, ${ }^{7-9}$ the viscoplastic strain rate is defined as

$$
\dot{\boldsymbol{\varepsilon}}^{\mathrm{vp}}=\eta<\phi(f)>\mathbf{n}
$$

in which $\eta$ is a fluidity parameter. The notation $<>$ refers to $\langle y\rangle=y H(y)$, where $H$ is the Heaviside step function. $\phi(f)$ is an arbitrary positive function of the yield function, for instance, a power law

$$
\phi(f)=\left[\frac{f}{\bar{\sigma}_{0}}\right]^{N}
$$

with $N$ a constant and $\bar{\sigma}_{0}$ the initial yield stress.

\subsection{Duvaut-Lions viscoplastic model}

A different viscoplastic model, which in its elaboration more closely connects to rate-independent plasticity, has been proposed by Duvaut and Lions. ${ }^{10,11,3}$ The theory is based on the difference in response between the rate-independent material and the viscoplastic material. The viscoplastic strain rate and the hardening law are defined as

$$
\begin{aligned}
\dot{\boldsymbol{\varepsilon}}^{\mathrm{vp}} & =\frac{1}{\zeta}\left[\mathbf{D}_{\mathrm{e}}\right]^{-1}(\boldsymbol{\sigma}-\overline{\boldsymbol{\sigma}}) \\
\dot{\kappa} & =-\frac{1}{\zeta}(\kappa-\bar{\kappa})
\end{aligned}
$$

where $\zeta$ is the relaxation time, $\overline{\boldsymbol{\sigma}}$ is the contribution of the rate-independent material ( we use a bar to denote the variable of the inviscid plastic model or back-bone model) which can be viewed as a projection of the current stress on the yield surface. The viscoplastic strain rate is determined by the difference between the total stress and the stress in the inviscid backbone model. This is in contrast with the Perzyna model in which the value of the yield surface $f$ determines the viscoplastic strain rate. The Duvaut-Lions model has the far-reaching advantage that it can be combined with a yield surface which has an apex (Drucker-Prager, Mohr-Coulomb) or which is non-smooth (Mohr-Coulomb, Tresca).

\subsection{Consistency model}

In the Perzyna and Duvaut-Lions viscoplastic theories an important distinction from the inviscid plasticity theory stems from the fact that the current stress states can be outside the yield surface and that the yield function may have a value larger than zero. Therefore, the Kuhn-Tucker conditions are not applicable. If the external loading remains constant the stresses return to the yield surface as a function of time. Because of this feature viscoplastic theories are commonly called overstress laws. We now consider another approach in which the strain-rate contribution (viscosity) is implemented through a rate-dependent yield surface. By doing so the Kuhn-Tucker conditions (9) remain valid and therefore we call this approach the consistency 
method. The yield function and the consistency condition for a rate-dependent material can be written as

$$
f(\boldsymbol{\sigma}, \kappa, \dot{\kappa})=0
$$

and

$$
\dot{f}(\boldsymbol{\sigma}, \kappa, \dot{\kappa})=\mathbf{n}: \dot{\boldsymbol{\sigma}}-h \dot{\kappa}-\xi \ddot{\kappa}=0
$$

where $\mathbf{n}$ is the gradient to the yield surface, $h$ the hardening modulus and $\xi$ the viscosity defined as follows:

$$
\mathbf{n}=\frac{\partial f}{\partial \boldsymbol{\sigma}}, \quad h=-\frac{\partial f}{\partial \kappa}, \quad \xi=-\frac{\partial f}{\partial \dot{\kappa}}
$$

In the consistency approach the yield surface can expand and shrink not only by hardening or softening effects, but also by hardening or softening rate effects. This has the advantage that the consistency method can describe the so-called Portevin-Le Chatelier effects in a proper way (S-type instability), which are caused by strain-rate softening (i.e. $h>0, \xi<0^{13}$ ).

\section{ALGORITHMIC ASPECTS}

In displacement-based finite element formulations, stress updates take place at the Gauss points for a prescribed nodal displacement. We start from time $t$ with the known converged state: $\left[\varepsilon_{t}, \varepsilon_{t}^{\mathrm{vp}}\right.$, $\left.\boldsymbol{\sigma}_{t}, \kappa_{t}\right]$ in a configuration $B_{t}$, to calculate the corresponding values at time $t+\Delta t:\left[\boldsymbol{\varepsilon}_{t+\Delta t}, \boldsymbol{\varepsilon}_{t+\Delta t}^{\mathrm{vp}}\right.$, $\left.\sigma_{t+\Delta t}, \kappa_{t+\Delta t}\right]$ in the updated configuration $B_{t+\Delta t}$, with a prescribed incremental nodal displacement $\Delta \mathbf{a}$ defining the geometric update $B_{t} \rightarrow B_{t+\Delta t}$. In this incremental process, the incremental strain $\Delta \varepsilon$ is decomposed into an elastic part $\Delta \varepsilon^{\mathrm{e}}$ and a viscoplastic part $\Delta \boldsymbol{\varepsilon}^{\mathrm{vp}}$ according to

$$
\Delta \varepsilon=\Delta \varepsilon^{\mathrm{e}}+\Delta \varepsilon^{\mathrm{vp}}
$$

The incremental stress is related to the elastic response by

$$
\Delta \boldsymbol{\sigma}=\mathbf{D}_{\mathrm{e}}\left(\Delta \varepsilon-\Delta \varepsilon^{\mathrm{vp}}\right)
$$

where, for algorithmic convenience, we have shifted to matrix vector notation. Therefore, the key feature of stress updates is characterized by estimating the incremental viscoplastic strain $\Delta \varepsilon^{\mathrm{vp}}$. We can use the generalized trapezoidal rule or the generalized midpoint rule to approximate the viscoplastic flow. The first algorithm is given by

$$
\Delta \varepsilon^{\mathrm{vp}}=\Delta \lambda\left[(1-\theta) \mathbf{n}_{t}+\theta \mathbf{n}_{t+\Delta t}\right], \quad \Delta \kappa=\Delta \lambda
$$

while the second method reads

$$
\Delta \varepsilon^{\mathrm{vp}}=\Delta \lambda \mathbf{n}_{t+\theta \Delta t}, \quad \Delta \kappa=\Delta \lambda
$$

where Von Mises plasticity is assumed and $\theta$ is an interpolation parameter such that $0 \leqslant \theta \leqslant 1$. For the one-step methods in this paper we adopt a generalized Euler method in which the 


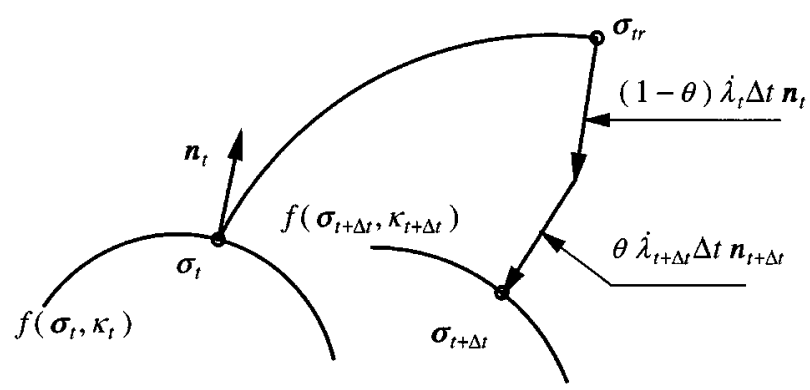

Figure 1. Geometric interpretation of the generalised Euler rule

viscoplastic flow is approximated as

$$
\begin{aligned}
\Delta \boldsymbol{\varepsilon}^{\mathrm{vp}} & =\left[(1-\theta) \dot{\boldsymbol{\varepsilon}}_{t}^{\mathrm{vp}}+\theta \dot{\boldsymbol{\varepsilon}}_{t+\Delta t}^{\mathrm{vp}}\right] \Delta t \\
\Delta \kappa & =\left[(1-\theta) \dot{\kappa}_{t}+\theta \dot{\kappa}_{t+\Delta t}\right] \Delta t
\end{aligned}
$$

The geometric interpretation of the Euler integration scheme is plotted in Figure 1. We note that for $\theta=0$ (explicit method) and $\theta=1$ (fully implicit method) the above three integration schemes are identical.

\subsection{Perzyna viscoplastic model}

In the one-step Euler integration scheme, the viscoplastic strain rate at the end of the time interval is expressed in a limited Taylor series expansion as ${ }^{4}$

$$
\dot{\boldsymbol{\varepsilon}}_{t+\Delta t}^{\mathrm{vp}}=\dot{\boldsymbol{\varepsilon}}_{t}^{\mathrm{vp}}+\left[\frac{\partial \dot{\boldsymbol{\varepsilon}}^{\mathrm{vp}}}{\partial \boldsymbol{\sigma}}\right]_{t} \Delta \boldsymbol{\sigma}+\left[\frac{\partial \dot{\boldsymbol{\varepsilon}}^{\mathrm{vp}}}{\partial \kappa}\right]_{t} \Delta \kappa=\dot{\boldsymbol{\varepsilon}}_{t}^{\mathrm{vp}}+\mathbf{G}_{t} \Delta \boldsymbol{\sigma}+\mathbf{h}_{t} \Delta \kappa
$$

where

$$
\mathbf{G}_{t}=\eta\left[\frac{\partial \phi}{\partial \boldsymbol{\sigma}} \mathbf{n}^{\mathrm{T}}+\phi \frac{\partial^{2} f}{\partial \boldsymbol{\sigma}^{2}}\right]_{t}, \quad \mathbf{h}_{t}=\eta\left[\frac{\partial \phi}{\partial \kappa} \mathbf{n}\right]_{t}
$$

and equations (11) and (14) have been applied. Substitution of equation (27) into equation (25) yields

$$
\Delta \boldsymbol{\varepsilon}^{\mathrm{vp}}=\left(\dot{\boldsymbol{\varepsilon}}_{t}^{\mathrm{vp}}+\theta \mathbf{G}_{t} \Delta \boldsymbol{\sigma}+\theta \mathbf{h}_{t} \Delta \kappa\right) \Delta t
$$

This equation can be substituted in the incremental stress-strain relation (22), which leads to

$$
\Delta \boldsymbol{\sigma}=\mathbf{D}_{\mathrm{c}} \Delta \boldsymbol{\varepsilon}-\Delta q
$$

in which

$$
\begin{aligned}
& \mathbf{D}_{\mathrm{c}}=\left[\mathbf{D}_{\mathrm{e}}^{-1}+\theta \Delta t \mathbf{G}_{t}\right]^{-1} \\
& \Delta q=\mathbf{D}_{\mathrm{c}}\left(\dot{\boldsymbol{\varepsilon}}_{t}^{\mathrm{vp}} \Delta t+\theta \Delta t \mathbf{h}_{t} \Delta \kappa\right)
\end{aligned}
$$


Box 1. A one-step Euler stress-update algorithm for Perzyna model

$$
\begin{aligned}
& \Delta \boldsymbol{\varepsilon}_{t+\Delta t}=\mathbf{B} \Delta \mathbf{a}_{t+\Delta t} \\
& \boldsymbol{\sigma}_{t r}=\boldsymbol{\sigma}_{t}+\mathbf{D}_{\mathrm{e}} \Delta \boldsymbol{\varepsilon}_{t+\Delta t} \\
& \text { If } f\left(\boldsymbol{\sigma}_{t r}, \kappa_{t}\right) \geqslant 0: \text { plastic state } \\
& \text { (1) } \mathbf{G}_{t}=\eta\left[\frac{\partial \phi}{\partial \boldsymbol{\sigma}} \mathbf{n}^{\mathrm{T}}+\phi \frac{\partial^{2} f}{\partial \boldsymbol{\sigma}^{2}}\right]_{t} \\
& \text { (2) } \mathbf{h}_{t}=\eta\left[\frac{\partial \phi}{\partial \kappa} \mathbf{n}\right]_{t} \\
& \text { (3) } \mathbf{D}_{\mathrm{c}}=\left[\mathbf{D}_{\mathrm{e}}^{-1}+\theta \Delta t \mathbf{G}_{t}\right]^{-1} \\
& \text { (4) } \Delta \mathbf{q}=\mathbf{D}_{\mathrm{c}}\left(\dot{\boldsymbol{\varepsilon}}_{t}^{\mathrm{vp}} \Delta t+\theta \Delta t \mathbf{h}_{t} \Delta \kappa^{(I)}\right)^{*} \\
& \text { (5) } \boldsymbol{\sigma}_{t+\Delta t}=\boldsymbol{\sigma}_{t}+\mathbf{D}_{\mathbf{c}} \Delta \boldsymbol{\varepsilon}_{t+\Delta t}-\Delta \mathbf{q} \\
& \text { (6) } f=f\left(\boldsymbol{\sigma}_{t+\Delta t}, \kappa_{t}+\Delta \kappa^{(I)}\right) \\
& \text { (7) } \dot{\boldsymbol{\varepsilon}}^{\mathrm{vp}}=\eta \phi\left(\boldsymbol{\sigma}_{t+\Delta t}, \kappa_{t}+\Delta \kappa\right) \frac{\partial f}{\partial \boldsymbol{\sigma}} \\
& \text { (8) } \dot{\kappa}_{t+\Delta t}=\sqrt{\frac{2}{3} \dot{\boldsymbol{\varepsilon}}^{\mathrm{vp}}: \dot{\boldsymbol{\varepsilon}}^{\mathrm{vp}}} \\
& \text { (9) } \Delta \kappa^{(I+1)}=\left[(1-\theta) \dot{\kappa}_{t}+\theta \dot{\kappa}_{t+\Delta t}\right] \Delta t \\
& \text { else elastic state : } \boldsymbol{\sigma}_{t+\Delta t}=\boldsymbol{\sigma}_{t r} \\
& \text { * (I) } \text { global iteration. }
\end{aligned}
$$

$\mathbf{D}_{\mathbf{c}}$ is the tangent stiffness matrix and $\Delta q$ gives the contribution of an extra pseudo-nodal force in the equation of motion. The Euler stress-update algorithm for Perzyna viscoplasticity is outlined in Box 1.

In the one-step Euler integration scheme, the current viscoplastic flow is estimated by a limited Taylor series expansion which depends on the gradient of the yield surface at time $t$. In a fully implicit integration scheme, the viscoplastic flow is determined by the gradient of the yield surface at time $t+\Delta t$. The incremental viscoplastic strain now reads

$$
\Delta \boldsymbol{\varepsilon}^{\mathrm{vp}}=\dot{\boldsymbol{\varepsilon}}_{t+\Delta t}^{\mathrm{vp}} \Delta t=\eta<\phi_{t+\Delta t}>\Delta t \mathbf{n}_{t+\Delta t}=\Delta \lambda \mathbf{n}_{t+\Delta t}
$$

where a plastic multiplier $\Delta \lambda$ is introduced under the constraint that the residual ${ }^{14}$

$$
r=\frac{\Delta \lambda}{\eta \Delta t}-\phi\left(\sigma_{t+\Delta t}, \lambda_{t+\Delta t}\right)
$$

goes to zero during the local iterative procedure. Substituting equation (32) into equation (22) yields

$$
\Delta \boldsymbol{\sigma}=\mathbf{D}_{\mathrm{e}}\left[\Delta \boldsymbol{\varepsilon}-\Delta \lambda \mathbf{n}_{t+\Delta t}\right]
$$


To compute $\Delta \lambda$, a local Newton-Raphson iteration process is applied. The iterative improvements of $\Delta \boldsymbol{\sigma}, \Delta \boldsymbol{\varepsilon}$ and $\Delta \lambda$ are denoted by $\delta \boldsymbol{\sigma}, \delta \boldsymbol{\varepsilon}$ and $\delta \lambda$, respectively. Differentiation of equation (34) gives the variation of $\Delta \boldsymbol{\sigma}$ as a function of the variation in $\Delta \varepsilon$ and $\Delta \lambda$ during iteration $i$ :

$$
\delta \boldsymbol{\sigma}=\mathbf{H} \delta \boldsymbol{\varepsilon}-\mathbf{H}\left[\frac{\partial f}{\partial \boldsymbol{\sigma}}+\Delta \lambda^{(i)} \frac{\partial^{2} f}{\partial \boldsymbol{\sigma} \partial \lambda}\right] \delta \lambda
$$

with a pseudo-elastic material stiffness matrix $\mathbf{H}$

$$
\mathbf{H}^{-1}=\mathbf{D}_{\mathrm{e}}^{-1}+\Delta \lambda^{(i)} \frac{\partial^{2} f}{\partial \boldsymbol{\sigma}^{2}}
$$

By differentiation of equation (33) the Newton-Raphson process at iteration $i+1$ is then described by

$$
\left(\frac{\partial \phi}{\partial \boldsymbol{\sigma}}\right)^{\mathrm{T}} \delta \boldsymbol{\sigma}+\left(\frac{\partial \phi}{\partial \lambda}-\frac{1}{\eta \Delta t}\right) \delta \lambda=-r^{(i)}
$$

with the residual $r^{(i)}$. Substitution of equation (35) into equation (37) yields

$$
\delta \lambda=\frac{1}{\alpha}\left[\left(\frac{\partial \phi}{\partial \boldsymbol{\sigma}}\right)^{\mathrm{T}} \mathbf{H} \delta \boldsymbol{\varepsilon}+r^{(i)}\right]
$$

with

$$
\alpha=\left(\frac{\partial \phi}{\partial \boldsymbol{\sigma}}\right)^{\mathrm{T}} \mathbf{H}\left[\frac{\partial f}{\partial \boldsymbol{\sigma}}+\Delta \lambda^{(i)} \frac{\partial^{2} f}{\partial \boldsymbol{\sigma} \partial \lambda}\right]+\frac{1}{\eta \Delta t}-\frac{\partial \phi}{\partial \lambda}
$$

Substitution of equation (38) into equation (35) subsequently leads to the tangent stiffness matrix

$$
\mathbf{D}_{\mathrm{c}}=\mathbf{H}-\frac{1}{\alpha} \mathbf{H}\left[\frac{\partial f}{\partial \boldsymbol{\sigma}}+\Delta \lambda^{(i)} \frac{\partial^{2} f}{\partial \boldsymbol{\sigma} \partial \lambda}\right]\left(\frac{\partial \phi}{\partial \boldsymbol{\sigma}}\right)^{\mathrm{T}} \mathbf{H}
$$

If we use local iterations during a global iteration, the iterative strain increment $\delta \boldsymbol{\varepsilon}$ vanishes from equation (38) due to a fixed total strain increment. In Box 2 the iterative implicit stress-update algorithm for Perzyna viscoplasticity is outlined.

To investigate the influence of the interpolation parameter $\theta$ on the approximation of the plastic flow, a unit cell test has been carried out, Figure 2. A four-noded element with a one-point Gauss integration scheme has been used. We take a consistent mass matrix and a constant time step. In Figure 3 the results for a constant velocity test (left) and a constant acceleration test (right) have been plotted. We observe that the results for a constant velocity test with different values for the interpolation parameter $\theta$ are identical after a few steps of plastic deformation. This is logical since the viscoplastic strain rate becomes constant due to the imposed constant velocity. For the constant acceleration test, the load-carrying capacity decreases with increasing values for the interpolation parameter $\theta$. It is noted that the results of the one-step Euler backward method $(\theta=1)$ and the iterative implicit method do not differ significantly even for the constant acceleration test, but this may change for larger time steps. 
Box 2. An iterative implicit stress-update algorithm for Perzyna model

$$
\begin{aligned}
& \Delta \boldsymbol{\varepsilon}_{t+\Delta t}=\mathbf{B} \Delta \mathbf{a}_{\mathbf{t}+\Delta t} \\
& \boldsymbol{\sigma}_{t r}=\boldsymbol{\sigma}_{t}+\mathbf{D}_{\mathbf{e}} \Delta \boldsymbol{\varepsilon}_{t+\Delta \mathrm{t}} \\
& \text { If } f\left(\boldsymbol{\sigma}_{t r}, \lambda_{t}\right) \geqslant 0 \text { : plastic state } \\
& \Delta \lambda^{(0)}=0, \quad \boldsymbol{\sigma}_{t+\Delta t}^{(0)}=\boldsymbol{\sigma}_{t}+\mathbf{D}_{\mathbf{e}}\left[\Delta \boldsymbol{\varepsilon}-\Delta \lambda^{(0)} \frac{\partial f}{\partial \boldsymbol{\sigma}}\right] \\
& r^{(0)}=\phi\left(\boldsymbol{\sigma}_{t+\Delta t}^{(0)}, \lambda_{t}+\Delta \lambda^{(0)}\right)-\frac{\Delta \lambda^{(0)}}{\eta \Delta t} \\
& \text { (1) local iteration } \\
& \text { (2) } \mathbf{H}=\left[\mathbf{D}_{\mathrm{e}}+\Delta \lambda^{(i)} \frac{\partial^{2} f}{\partial \boldsymbol{\sigma}^{2}}\right]^{-1} \\
& \text { (3) } \alpha=\left(\frac{\partial \phi}{\partial \boldsymbol{\sigma}}\right)^{\mathrm{T}} \mathbf{H}\left[\Delta \lambda^{(i)} \frac{\partial^{2} f}{\partial \boldsymbol{\sigma} \partial \lambda}+\frac{\partial f}{\partial \boldsymbol{\sigma}}\right]+\frac{1}{\eta \Delta t}-\frac{\partial \phi}{\partial \lambda} \\
& \text { (4) } \Delta \lambda^{(i+1)}=\Delta \lambda^{(i)}+\frac{r^{(i)}}{\alpha} \\
& \text { (5) } \boldsymbol{\sigma}_{t+\Delta t}^{(i+1)}=\boldsymbol{\sigma}_{t}+\mathbf{D}_{\mathbf{e}}\left[\Delta \boldsymbol{\varepsilon}-\Delta \lambda^{(i+1)} \frac{\partial f}{\partial \boldsymbol{\sigma}}\right] \\
& \text { (6) } r^{(i+1)}=\phi\left(\boldsymbol{\sigma}_{t+\Delta t}^{i+1)}, \lambda_{t}+\Delta \lambda^{(i+1)}\right)-\frac{\Delta \lambda^{(i+1)}}{\eta \Delta t} \\
& \text { (7) if }\left|r^{(i+1)}\right|>\delta \text { go to }(1) \\
& \text { else elastic state : } \boldsymbol{\sigma}_{t+\Delta t}=\boldsymbol{\sigma}_{t r}
\end{aligned}
$$

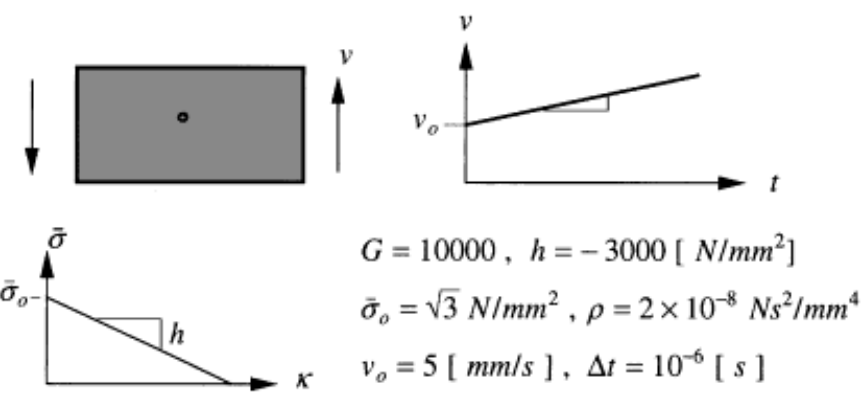

Figure 2. Unit cell under dynamic shear 

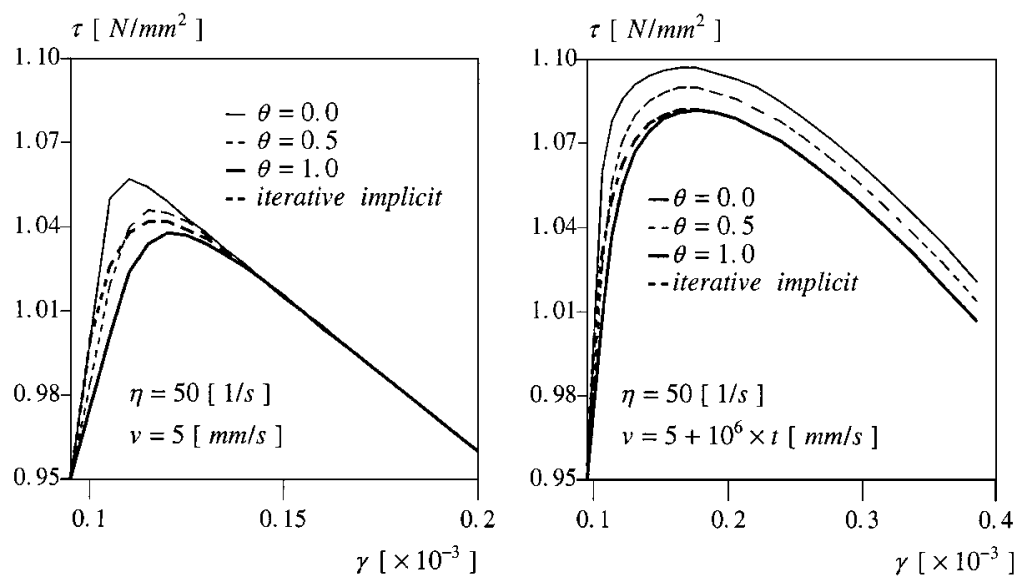

Figure 3. Unit cell—Perzyna model. Left: constant velocity. Right: constant acceleration

\subsection{Duvaut-Lions viscoplastic model}

In Duvaut-Lions viscoplastic model, the stress update is carried out in two steps. First the inviscid back-bone stress $\overline{\boldsymbol{\sigma}}$ is updated. An Euler backward algorithm is used for the return mapping of the stress to the yield surface

$$
\Delta \overline{\boldsymbol{\sigma}}=\overline{\mathbf{D}}_{\mathrm{c}} \Delta \boldsymbol{\varepsilon}
$$

where

$$
\overline{\mathbf{D}}_{\mathrm{c}}=\mathbf{D}_{\mathrm{e}}-\frac{\mathbf{D}_{\mathrm{e}} \overline{\mathbf{n}} \overline{\mathbf{n}}^{\mathrm{T}} \mathbf{D}_{\mathrm{e}}}{h+\overline{\mathbf{n}}^{\mathrm{T}} \mathbf{D}_{\mathrm{e}} \overline{\mathbf{n}}}
$$

and

$$
\overline{\mathbf{n}}=\frac{\partial f}{\partial \overline{\boldsymbol{\sigma}}}
$$

Next, the viscoplastic response is determined. The current viscoplastic strain rate $\dot{\varepsilon}_{t+\Delta t}^{\mathrm{vp}}$ can be expressed as

$$
\dot{\boldsymbol{\varepsilon}}_{t+\Delta t}^{\mathrm{vp}}=\frac{1}{\zeta} \mathbf{D}_{\mathrm{e}}^{-1}\left(\boldsymbol{\sigma}_{t+\Delta t}-\overline{\boldsymbol{\sigma}}_{t+\Delta t}\right)=\frac{1}{\zeta} \mathbf{D}_{\mathrm{e}}^{-1}\left(\Delta \boldsymbol{\sigma}-\Delta \overline{\boldsymbol{\sigma}}+\boldsymbol{\sigma}_{t}^{\mathrm{vp}}\right)
$$

where $\boldsymbol{\sigma}_{t}^{\mathrm{vp}}=\boldsymbol{\sigma}_{t}-\overline{\boldsymbol{\sigma}}_{t}$. Now the viscoplastic strain increment $\Delta \boldsymbol{\varepsilon}^{\mathrm{vp}}$ in equation (25) becomes

$$
\Delta \boldsymbol{\varepsilon}^{\mathrm{vp}}=\left[(1-\theta) \dot{\boldsymbol{\varepsilon}}_{t}^{\mathrm{vp}}+\frac{\theta}{\zeta} \mathbf{D}_{\mathrm{e}}^{-1}\left(\Delta \boldsymbol{\sigma}-\Delta \overline{\boldsymbol{\sigma}}+\boldsymbol{\sigma}_{t}^{\mathrm{vp}}\right)\right] \Delta t
$$

Substitution of equation (45) into equation (22) yields

$$
\Delta \boldsymbol{\sigma}=\mathbf{D}_{\mathrm{c}} \Delta \boldsymbol{\varepsilon}-\Delta q
$$


Box 3. A one-step implicit stress-update algorithm for DuvautLions model
$\Delta \boldsymbol{\varepsilon}_{t+\Delta t}=\mathbf{B} \Delta \mathbf{a}_{t+\Delta t}$
$\boldsymbol{\sigma}_{t r}=\boldsymbol{\sigma}_{t}+\mathbf{D}_{\mathbf{e}} \Delta \varepsilon_{t+\Delta t}$
If $f\left(\boldsymbol{\sigma}_{t r}, \kappa_{t}\right) \geqslant 0$ : plastic state
(1) $\overline{\boldsymbol{\sigma}}_{t+\Delta t}=\overline{\boldsymbol{\sigma}}_{t}+\overline{\mathbf{D}}_{\mathrm{c}} \Delta \boldsymbol{\varepsilon}$
(2) $\bar{\kappa}_{t+\Delta t}=\bar{\kappa}_{t}+\dot{\bar{\kappa}}_{t+\Delta t} \Delta t$
(3) $\mathbf{D}_{\mathbf{c}}=\frac{\zeta}{\zeta+\theta \Delta t}\left[D_{\mathrm{e}}+\frac{\theta \Delta t}{\zeta} \overline{\mathbf{D}}_{\mathrm{c}}\right]$
(4) $\Delta \mathbf{q}=\frac{\zeta \Delta t}{\zeta+\theta \Delta t}\left[(1-\theta) \mathbf{D}_{\mathbf{e}} \dot{\boldsymbol{\varepsilon}}_{t}^{\mathrm{vp}}+\frac{\theta}{\zeta}\left(\boldsymbol{\sigma}_{t}-\overline{\boldsymbol{\sigma}}_{t}\right)\right]$
(5) $\boldsymbol{\sigma}_{t+\Delta t}=\boldsymbol{\sigma}_{t}+\mathbf{D}_{\mathbf{c}} \Delta \boldsymbol{\varepsilon}-\Delta \mathbf{q}$
(6) $\dot{\boldsymbol{\varepsilon}}_{t+\Delta t}^{\mathrm{vp}}=\frac{1}{\zeta} \mathbf{D}_{\mathrm{e}}^{-1}\left(\boldsymbol{\sigma}_{t+\Delta t}-\overline{\boldsymbol{\sigma}}_{t+\Delta t}\right)$
(7) $\dot{\kappa}_{t+\Delta t}=\sqrt{\left(\dot{\boldsymbol{\varepsilon}}_{t+\Delta t}^{\mathrm{vp}}\right)^{\mathrm{T}} A \dot{\boldsymbol{\varepsilon}}_{t+\Delta t}^{\mathrm{vp}}}, \quad A=\operatorname{diag}\left[\begin{array}{llllll}\frac{2}{3} & \frac{2}{3} & \frac{2}{3} & \frac{1}{3} & \frac{1}{3} & \frac{1}{3}\end{array}\right]$
(8) $\kappa_{t+\Delta t}=\bar{\kappa}_{t+\Delta t}-\zeta \dot{\kappa}_{t+\Delta t}$
else elastic state : $\boldsymbol{\sigma}_{t+\Delta t}=\boldsymbol{\sigma}_{t r}$

in which

$$
\mathbf{D}_{\mathrm{c}}=\frac{\zeta}{\zeta+\theta \Delta t}\left[\mathbf{D}_{\mathrm{e}}+\frac{\theta \Delta t}{\zeta} \overline{\mathbf{D}}_{\mathrm{c}}\right], \quad \Delta q=\frac{\zeta \Delta t}{\zeta+\theta \Delta t}\left[(1-\theta) \mathbf{D}_{\mathrm{e}} \dot{\mathbf{\varepsilon}}_{t}^{\mathrm{vp}}+\frac{\theta}{\zeta} \boldsymbol{\sigma}_{t}^{\mathrm{vp}}\right]
$$

where $\Delta q$ represents the contribution of an extra pseudo-nodal force in the equation of motion. Note that with $\theta=1$ the tangent $\mathbf{D}_{\mathbf{c}}$ for the backward Euler algorithm as $\mathrm{in}^{15}$ is recovered. In Box 3 the stress-update algorithm for Duvaut-Lions viscoplasticity is outlined.

\subsection{Consistency model}

In the consistency approach the plastic flow direction is defined in the same way as in the Perzyna model [cf. equation (14)]

$$
\Delta \boldsymbol{\varepsilon}^{\mathrm{vp}}=\Delta \lambda \mathbf{n}
$$

The plastic multiplier is now determined by the discretized consistency condition of the yield surface

$$
f(\boldsymbol{\sigma}, \lambda, \dot{\lambda}) \approx f^{(i)}+\mathbf{n}^{\mathrm{T}} \delta \boldsymbol{\sigma}+\frac{\partial f}{\partial \lambda} \delta \lambda+\frac{\partial f}{\partial \dot{\lambda}} \delta \dot{\lambda}=0
$$


where $f^{(i)}=f\left(\sigma^{(i)}, \lambda^{(i)}, \dot{\lambda}^{(i)}\right)$ is the $i$ th residual of the yield function. For algorithmic convenience, we directly express the yield surface as a function of plastic multiplier since $\dot{\kappa}=\dot{\lambda}$ for Von Mises plasticity. We again use a local Newton-Raphson iteration process to compute the plastic multiplier $\Delta \lambda$. The multiplier $\Delta \lambda$ is integrated by an Euler backward algorithm

$$
\delta \lambda^{(i)}=\delta \dot{\lambda} \Delta t, \quad \Delta \lambda^{(i)}=\sum_{i} \delta \lambda^{(i)}
$$

Accordingly, equation (49) can be rewritten as

$$
\mathbf{n}^{\mathrm{T}} \delta \boldsymbol{\sigma}+\left[\frac{\partial f}{\partial \lambda}+\frac{1}{\Delta t} \frac{\partial f}{\partial \dot{\lambda}}\right] \delta \lambda=-f^{(i)}
$$

Substitution of equation (48) into equation (22), differentiation and use of equation (50) leads to

$$
\delta \boldsymbol{\sigma}=\mathbf{H} \delta \boldsymbol{\varepsilon}-\mathbf{H}\left[\frac{\partial f}{\partial \boldsymbol{\sigma}}+\Delta \lambda^{(i)} \frac{\partial^{2} f}{\partial \boldsymbol{\sigma} \partial \lambda}+\frac{\Delta \lambda^{(i)}}{\Delta t} \frac{\partial^{2} f}{\partial \boldsymbol{\sigma} \partial \dot{\lambda}}\right] \delta \lambda
$$

where $\mathbf{H}$ is defined as in equation (36). Elimination of $\delta \boldsymbol{\sigma}$ by substitution of equation (52) into equation (51) yields

$$
\delta \lambda=\frac{1}{\beta}\left(\mathbf{n}^{\mathrm{T}} \mathbf{H} \delta \boldsymbol{\varepsilon}+f^{(i)}\right)
$$

with

$$
\beta=\mathbf{n}^{\mathrm{T}} \mathbf{H}\left[\frac{\partial f}{\partial \boldsymbol{\sigma}}+\Delta \lambda^{(i)} \frac{\partial^{2} f}{\partial \boldsymbol{\sigma} \partial \lambda}+\frac{\Delta \lambda^{(i)}}{\Delta t} \frac{\partial^{2} f}{\partial \boldsymbol{\sigma} \partial \dot{\lambda}}\right]-\frac{\partial f}{\partial \lambda}-\frac{1}{\Delta t} \frac{\partial f}{\partial \dot{\lambda}}
$$

Substitution of equation (53) into equation (52) gives the tangent stiffness matrix

$$
\mathrm{D}_{\mathrm{c}}=\mathbf{H}-\frac{1}{\beta} \mathbf{H}\left[\frac{\partial f}{\partial \boldsymbol{\sigma}}+\Delta \lambda^{(i)} \frac{\partial^{2} f}{\partial \boldsymbol{\sigma} \partial \lambda}+\frac{\Delta \lambda^{(i)}}{\Delta t} \frac{\partial^{2} f}{\partial \boldsymbol{\sigma} \partial \dot{\lambda}}\right] \mathbf{n}^{\mathrm{T}} \mathbf{H}
$$

Again, if we use local iterations during a global iteration, the iterative strain increment $\delta \boldsymbol{\varepsilon}$ vanishes from equation (53). The stress-update algorithm for the consistency model is outlined in Box 4.

\section{VISCOPLASTICITY AND H-TYPE AND S-TYPE INSTABILITIES}

To compare the three viscoplastic models in a quantitative context, we consider a simple shear problem $\left(2 \dot{\varepsilon}_{x y}=\dot{\gamma}=\partial v / \partial x, \sigma_{x y}=\tau\right)$ with a Von Mises yield function

$$
f(\boldsymbol{\sigma}, \kappa)=\sqrt{3} \tau-\bar{\sigma}(\kappa)
$$

For the Perzyna model we apply equation (14) and equation (15) with $N=1$

$$
\dot{\gamma}^{\mathrm{vp}}=\frac{\eta}{\bar{\sigma}_{0}}[\sqrt{3} \tau-\bar{\sigma}(\kappa)] \sqrt{3}
$$


Box 4. A fully implicit stress-update algorithm for the consistency model

$\Delta \boldsymbol{\varepsilon}_{t+\Delta t}=\mathbf{B} \Delta \mathbf{a}_{t+\Delta t}$
$\boldsymbol{\sigma}_{t r}=\boldsymbol{\sigma}_{t}+\mathbf{D}_{\mathbf{e}} \Delta \boldsymbol{\varepsilon}_{t+\Delta t}$
if $f\left(\boldsymbol{\sigma}_{t r}, \lambda_{t, t}\right) \geqslant 0$ : plastic state
$\Delta \lambda^{(0)}=0, \quad \dot{\lambda}_{t+\Delta t}^{(0)}=\frac{\Delta \lambda^{(0)}}{\Delta t}, \quad \boldsymbol{\sigma}_{t+\Delta t}^{(0)}=\boldsymbol{\sigma}_{t}+\mathbf{D}_{\mathbf{e}}\left[\Delta \boldsymbol{\varepsilon}-\Delta \lambda^{(0)} \frac{\partial f}{\partial \boldsymbol{\sigma}}\right]$
$f^{(0)}=f\left(\boldsymbol{\sigma}_{t+\Delta t}^{(0)}, \lambda_{t}+\Delta \lambda^{(0)}, \dot{\lambda}_{t+\Delta t}^{(0)}\right)$
(1) local iteration
(2) $\mathbf{H}^{(i)}=\left[\mathbf{D}_{\mathrm{e}}+\Delta \lambda^{(i)} \frac{\partial^{2} f}{\partial \boldsymbol{\sigma}^{2}}\right]^{-1}$
(3) $\beta=\left(\frac{\partial f}{\partial \boldsymbol{\sigma}}\right)^{\mathrm{T}} \mathbf{H}\left[\frac{\partial f}{\partial \boldsymbol{\sigma}}+\Delta \lambda^{(i)} \frac{\partial^{2} f}{\partial \boldsymbol{\sigma} \partial \lambda}+\frac{\Delta \lambda^{(i)}}{\Delta t} \frac{\partial^{2} f}{\partial \boldsymbol{\sigma} \partial \dot{\lambda}}\right]-\frac{\partial f}{\partial \lambda}-\frac{1}{\Delta t} \frac{\partial f}{\partial \dot{\lambda}}$
(4) $\Delta \lambda^{(i+1)}=\Delta \lambda^{(i)}+\frac{f^{(i)}}{\beta}$
(5) $\boldsymbol{\sigma}_{t+\Delta t}^{(i+1)}=\boldsymbol{\sigma}_{t}+\mathbf{D}_{\mathrm{e}}\left[\Delta \boldsymbol{\varepsilon}-\Delta \lambda^{(i+1)} \frac{\partial f}{\partial \boldsymbol{\sigma}}\right]$
(6) $f^{(i+1)}=f\left(\boldsymbol{\sigma}_{t+\Delta t}^{(i+1)}, \lambda_{t}+\Delta \lambda^{(i+1)}, \dot{\lambda}_{t+\Delta t}^{(i+1)}\right)$
(7) if $\left|f^{(i+1)}\right|>\delta$ go to $(1)$
else elastic state: $\boldsymbol{\sigma}_{t+\Delta t}=\boldsymbol{\sigma}_{t r}$

After rewriting equation (57) we obtain

$$
\tau=\frac{\bar{\sigma}}{\sqrt{3}}+\left(\frac{\bar{\sigma}_{0}}{3 \eta}\right) \dot{\gamma}^{\mathrm{vp}}
$$

Differentiating equation (58) and substituting $\dot{\bar{\sigma}}=h \dot{\kappa}$ and $\dot{\kappa}=\dot{\gamma}^{\mathrm{vp}} / \sqrt{3}$ then results in

$$
\dot{\tau}=\frac{h}{3} \dot{\gamma}^{\mathrm{vp}}+\left(\frac{\bar{\sigma}_{0}}{3 \eta}\right) \ddot{\gamma}^{\mathrm{vp}}
$$

If we combine the constitutive equation (59) with the equations of motion (1), the kinematic equation (2) and take $\gamma^{\mathrm{vp}}=\dot{\gamma}-\dot{\tau} / G$, we obtain a third-order differential equation for the $1-\mathrm{D}$ Perzyna viscoplastic strain-softening element

$$
m\left(\frac{1}{c_{g}^{2}} \frac{\partial^{3} v}{\partial t^{3}}-\frac{\partial^{3} v}{\partial x^{2} \partial t}\right)+\frac{3 G+h}{c_{g}^{2}} \frac{\partial^{2} v}{\partial t^{2}}-h \frac{\partial^{2} v}{\partial x^{2}}=0 \quad \text { with } \quad m=\frac{\bar{\sigma}_{0}}{\eta}
$$


where $G$ is the shear modulus and $c_{g}=\sqrt{G / \rho}$ the elastic shear wave speed. If the viscosity parameter $m \rightarrow 0$ the classical rate-independent wave equation is recovered with imaginary wave speed in case of strain softening $(h<0) .{ }^{4}$ Furthermore, upon a decreasing loading rate the third-order terms in equation (60) become less dominant.

By means of an analysis of characteristics we obtain the characteristic determinant:

$$
D=m\left[\left(1 / c_{g}^{2}\right) \mathrm{d} x^{2}-\mathrm{d} t^{2}\right]
$$

with $D=0$ a curve in the $v-x-t$ plane which coincides with the characteristics $( \pm \mathrm{d} x / \mathrm{d} t)$ equal to the elastic shear wave speed $\pm c_{g}$. This means that the wave equation remains hyperbolic and the initial value problem is well-posed also after the onset of softening. An investigation of the strain rate distribution along a one-dimensional element has resulted into an implicit expression for the internal length scale $l:^{5}$

$$
\mathrm{e}^{-\frac{3 G l}{2 c_{g} m}}\left[1+\frac{4 h+3 G}{4 c_{g} m} l\right] \approx \beta
$$

where $\beta$ is a small constant which represents the cut-off value of the relative strain rate at the edge of the shear band. The derivation in Appendix I is based on high loading rates and for this reason the loading rate does not enter equation (62). However, for loading rates of several orders of magnitude smaller than considered in this paper equation (62) no longer holds. An examination of the influence of the imperfection has revealed that the width of the localization zone $L$ is determined by the smaller value of the internal length scale $l$ and the imperfection size $w^{5}$

$$
L=\min [l, w]
$$

Next, the Duvaut-Lions model is examined. For the case of simple shear equation (16) becomes

$$
\dot{\gamma}^{\mathrm{vp}}=\frac{1}{G \zeta}(\tau-\bar{\tau})
$$

If we use equation (64) in rate format and take $\dot{\bar{\tau}}=(h / \sqrt{3}) \dot{\bar{\gamma}}^{\mathrm{p}}$ we obtain

$$
\dot{\tau}=\frac{h}{\sqrt{3}} \dot{\bar{\gamma}}^{\mathrm{p}}+\zeta G \ddot{\gamma}^{\mathrm{vp}}
$$

where the Von Mises back-bone solid is assumed. Note that both $\bar{\gamma}^{\mathrm{p}}$ and $\gamma^{\mathrm{vp}}$ enter the equation which is different from the Perzyna model. Since

$$
\dot{\gamma}=\dot{\bar{\gamma}}^{\mathrm{e}}+\dot{\bar{\gamma}}^{\mathrm{p}}=\dot{\gamma}^{\mathrm{ve}}+\dot{\gamma}^{\mathrm{vp}}
$$

the back-bone stress can be written as

$$
\dot{\bar{\tau}}=\frac{h}{\sqrt{3}} \dot{\bar{\gamma}}^{\mathrm{p}}=\frac{h}{\sqrt{3}}\left(\dot{\gamma}-\frac{\dot{\bar{\tau}}}{G}\right)
$$


which can be rewritten in

$$
\dot{\bar{\tau}}=\frac{G(h / \sqrt{3})}{G+(h / \sqrt{3})} \dot{\gamma}
$$

If we combine the constitutive equations (65) and (68) with the equations of motion (1), the kinematic equation (2) and take $\gamma^{\mathrm{vp}}=\dot{\gamma}-\dot{\tau} / G$, we obtain the third-order differential equation for the one-dimensional Duvaut-Lions viscoplastic strain-softening bar in shear

$$
m\left(\frac{1}{c_{g}^{2}} \frac{\partial^{3} v}{\partial t^{3}}-\frac{\partial^{3} v}{\partial x^{2} \partial t}\right)+\frac{3 G+(\sqrt{3} h)}{c_{g}^{2}} \frac{\partial^{2} v}{\partial t^{2}}-(\sqrt{3} h) \frac{\partial^{2} v}{\partial x^{2}}=0 \quad \text { with } \quad m=\zeta(3 G+(\sqrt{3} h))
$$

This equation is similar to that derived for Perzyna viscoplasticity, equation (60). Therefore, the wave equation remains hyperbolic and the initial value problem is well-posed. If we replace $h$ in equation (62) by $\sqrt{3} h$ we obtain the expression for the width of the localization zone for the Duvaut-Lions model.

Finally, the consistency model is investigated. For the simple shear problem with a Von Mises yield function and a strain-hardening hypothesis $\left(\dot{\kappa}=\dot{\gamma}^{\mathrm{vp}} / \sqrt{3}\right)$, equation (19) leads to

$$
\dot{\tau}=\frac{h}{3} \dot{\gamma}^{\mathrm{vp}}+\frac{\xi}{3} \ddot{\gamma}^{\mathrm{vp}}
$$

The representation of a one-dimensional viscoplastic element is thus the same as the Perzyna model, cf. equation (59). The wave equation and the expression for the internal length-scale are obtained directly by setting $m=\xi$ in equations (60) and (62), respectively.

From the differential equations for the one-dimensional case we derived that the three different viscoplastic models have a regularizing effect in the sense that they keep the field equations hyperbolic and therefore the initial-value problem remains well-posed. By introducing a viscosity parameter $m$ :

$$
\begin{aligned}
\text { Perzyna model: } & m=\frac{\sigma_{0}}{\eta} \\
\text { Duvaut-Lions model: } & m=\zeta(3 G+(\sqrt{3} h)) \\
\text { Consistency model: } & m=\xi
\end{aligned}
$$

the three models have the same length scale equation (62) which specifies the width of the shear band.

Because for the one-dimensional case the three viscoplastic models can be cast into the same format by introducing a viscosity parameter $m$, we can compare them by choosing the material parameters such that they have the same viscosity parameter $m$. Figure 4 shows that for the unit cell in shear the results for the Perzyna model and the consistency approach are identical. The results for the Duvaut-Lions model are slightly different from the results for Perzyna and consistency models because we take the same softening parameter $h$. In the formulation for Duvaut-Lions we have $\sqrt{3} h$ which causes the difference, cf. equations (69) and (60). 

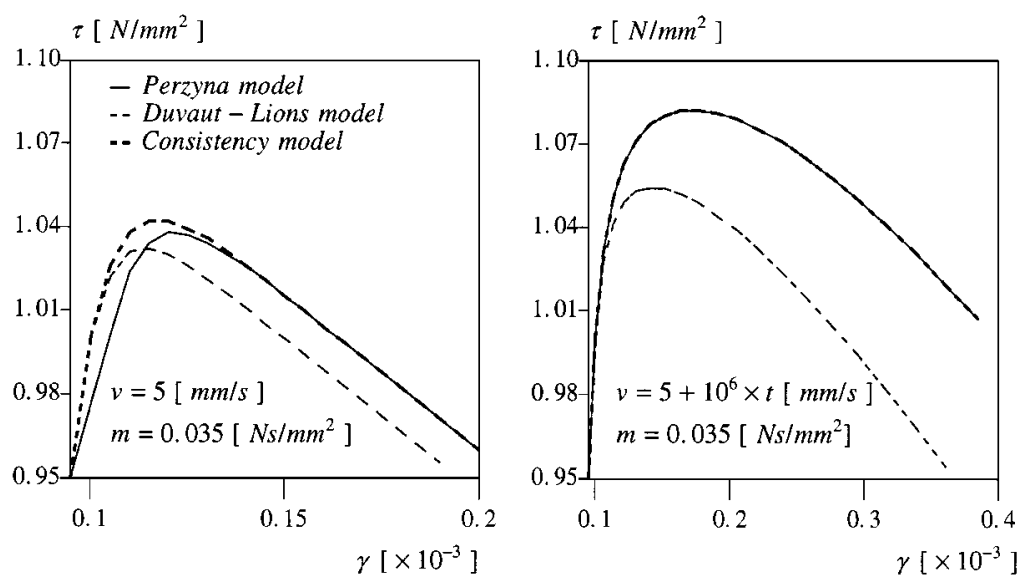

Figure 4. Unit cell—comparison of viscoplastic models. Left: constant velocity. Right: constant acceleration

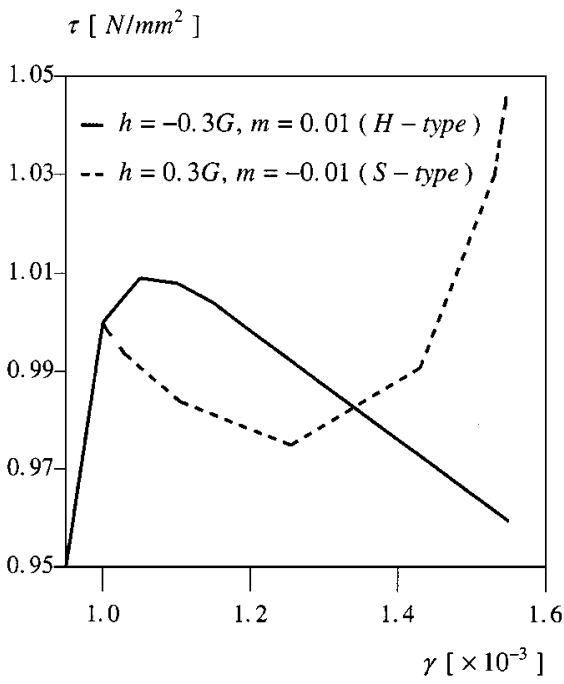

Figure 5. Unit cell under dynamic shear for H-type instability $(h<0, m>0)$ and S-type instability $(h>0, m<0)$

In the consistency model the yield surface can expand or shrink not only by hardening or softening effects, but also by hardening rate or softening rate effects. The competition of the strain and strain-rate influence can trigger so-called H-type instabilities (i.e. $h<0, m>0$ ) and so-called S-type instabilities (i.e. $h>0, m<0) .{ }^{12}$ In Figure 5 typical stress-strain relations for the occurrence of the H-type and the S-type instabilities are shown. For the H-type instabilities a stationary shear band will occur at the later stages of deformation. For the S-type instabilities, a Portevin-Le Chatelier (PLC) band occurs at the initial stages of deformation. Due to the contribution of strain hardening the PLC band will be arrested locally and a travelling PLC effect will be observed. 


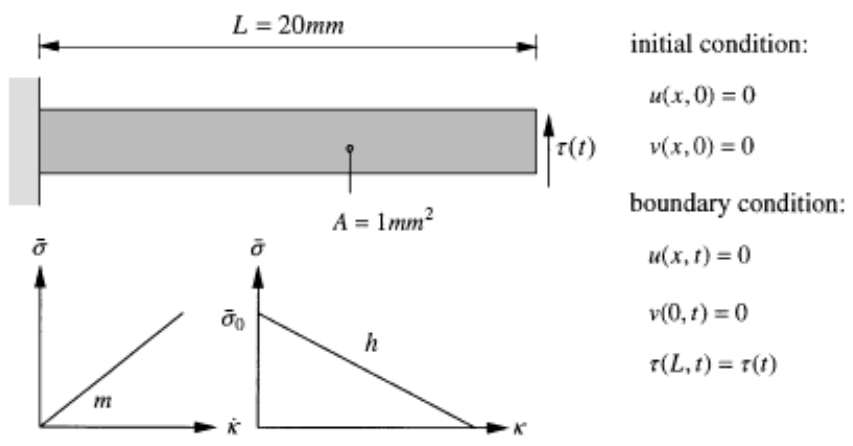

Figure 6. One-dimensional problem in shear-H-type instability

\section{NUMERICAL EXAMPLES}

To demonstrate the applicability of the computational procedures, finite element analyses have been carried out for a one-dimensional bar, Figure 6 . The bar is fixed at the left end and a shear stress is imposed at the right end. By keeping all horizontal displacements equal to zero, we simulate a pure one-dimensional shear problem. In all analyses, the spatial discretization was performed using eight-noded quadrilateral elements with a $3 \times 3$ Gauss integration scheme. We use a consistent mass matrix and an implicit time-integration scheme based on the trapezoidal rule. All calculations presented here have been carried out with a time step smaller than the critical time step $\Delta t_{\mathrm{cr}}=\Delta l / c_{g}$, where $\Delta l$ is the size of the smallest finite element.

\subsection{Shear layer: H-type instability}

The set-up of the problem is shown in Figure 6 together with the constitutive relations that give rise to an $\mathrm{H}$-type instability with strain-softening $(h<0)$ and strain-rate hardening $(m>0)$. First, the width of the shear band has been investigated for the three viscoplasticity models. The internal length scale results from equation (62) with the material parameters in the corresponding figures. The relative strain distributions have been plotted in Figure 7 at the time when the loading pulse has returned to the centre of the bar $\left(t=3 \times 10^{-5} \mathrm{~s}\right)$. We observe that upon mesh refinement, the shear band thickness converges to the material length scale $l$ as defined in equation (62). Clearly, the width of the shear band decreases when the viscosity $m$ decreases or when the softening modulus $h$ becomes more negative.

Next, the influence of a material imperfection is analysed. We take the imperfection sizes $w=1,2,4,8 \mathrm{~mm}$, respectively, at the left end of the shear layer by means of a 10 per cent reduction in yield strength. The relative strain distributions have been plotted in Figure 8 for the three viscoplasticity models at the time when the reflected stress wave has reached the centre $(x=10 \mathrm{~mm})$ of the bar. We observe that for all viscoplastic models, the imperfection size dominates the width of the shear band when the value for $w$ is smaller than the material length scale $l$. On the other hand, if the imperfection size is larger than the material length scale $(w>l)$, the influence of the imperfection disappears and the material length scale determines the width of the shear band. 
Finally, a comparison is presented between the three viscoplasticity models. We take the same softening modulus $h$ and the viscosity $m$ is chosen such that the internal length scales are approximately the same. In Figure 9 the distribution of the plastic strain is plotted. The results are almost identical for the three viscoplasticity models. We observe that the peak strain in the shear band for the Duvaut-Lions model is slightly larger than those for the Perzyna model and for the consistency model. It is finally noted that the consistency model shows a faster global convergence than the other models during plastic loading. Box 5 shows the average number of global iterations per loading step for the different models.
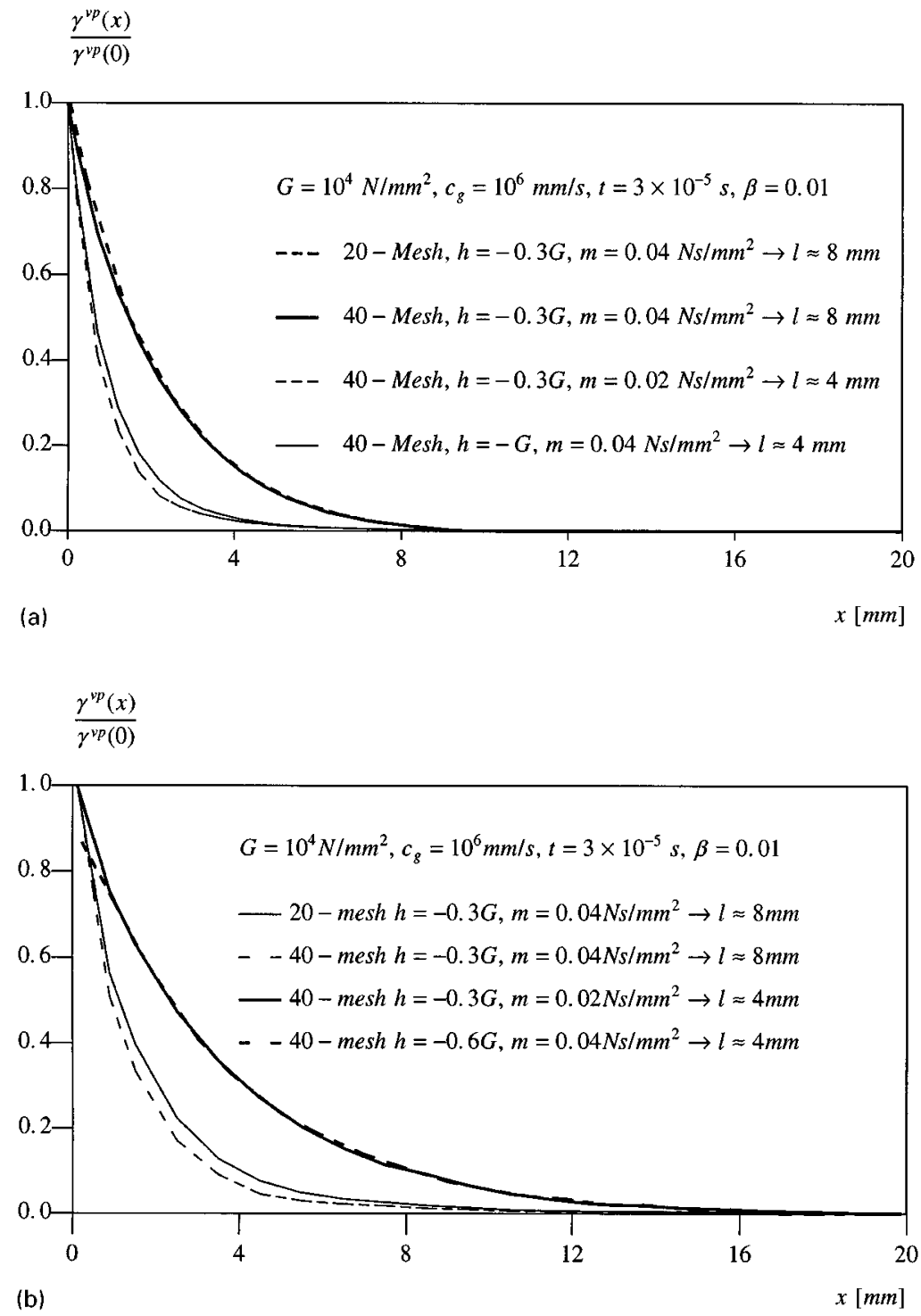

Figure 7. $(\mathrm{a}, \mathrm{b})$ 


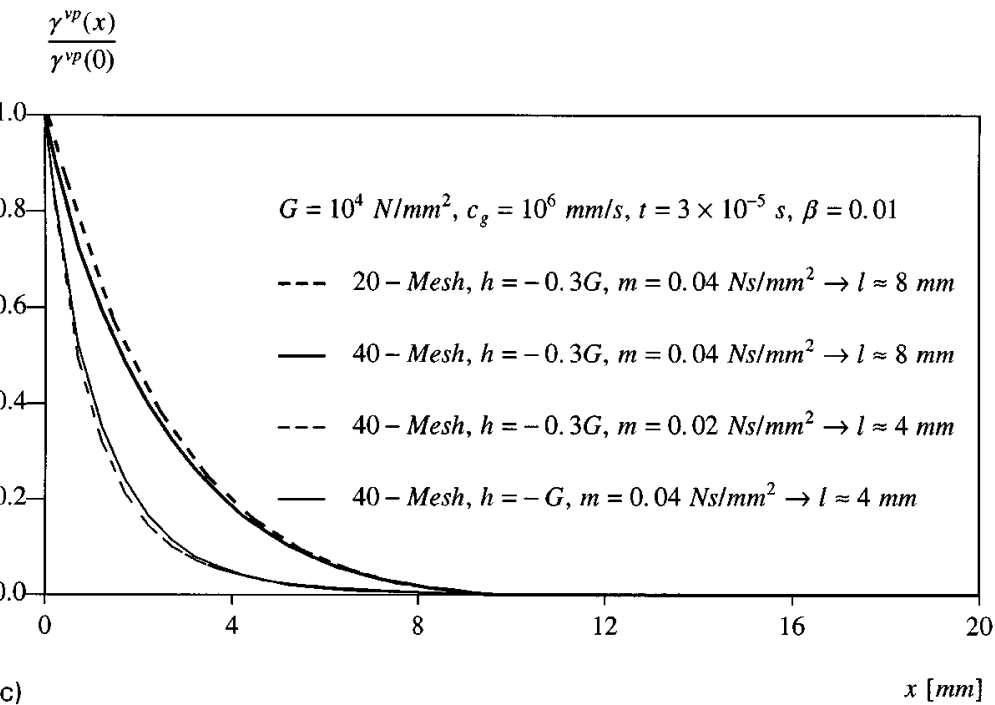

Figure 7. (a) Perzyna viscoplastic model: the width of the shear band upon variation of $m$ and $h$-Comparison with analytical solution for $l$ via equation (62). (b) Duvaut-Lions viscoplastic model: the width of the shear band upon variation of $m$ and $h$-Comparison with analytical solution for $l$ via equation (62). (c) Consistency viscoplastic model: the width of the shear band upon variation of $m$ and $h$-Comparison with analytical solution for $l$ via equation (62)

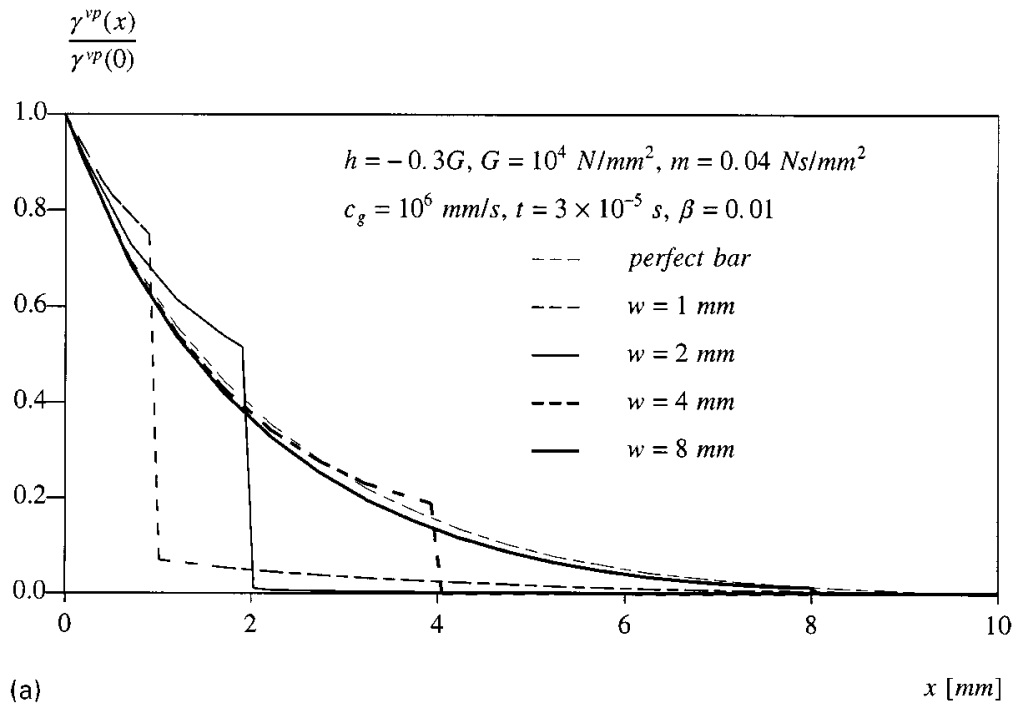

Figure 8(a) 

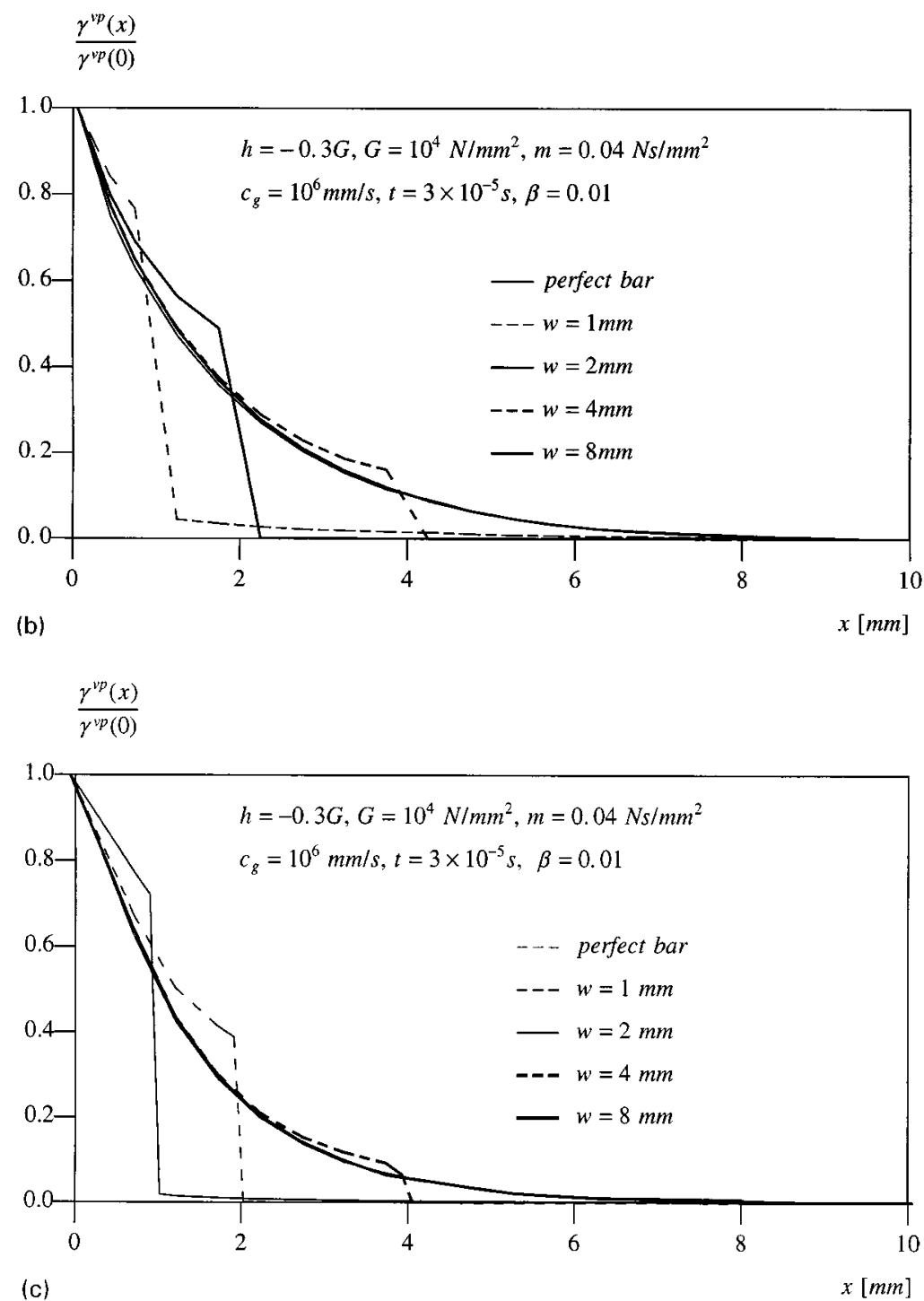

Figure 8. (a) The influence of an imperfection: Perzyna viscoplastic model $(l=8 \mathrm{~mm})$. (b) The influence of an imperfection: Duvaut-Lions viscoplastic model $(l=8 \mathrm{~mm})$. (c) The influence of an imperfection: consistency viscoplastic model $(l=8 \mathrm{~mm})$

\subsection{Shear layer: S-type instability}

The shear layer problem is now re-analysed with a different material law, Figure 10, that incorporates strain hardening $(h>0)$ and strain-rate softening $(m<0)$. This so-called S-type instability is different from an H-type instability (i.e. $h<0, m>0$ ) which produces a stationary shear band. Now, a propagative shear band is observed, which is commonly referred to as a Portevin-Le Chatelier (PLC) band. ${ }^{12}$ The competition between the strain hardening $(h>0)$ 


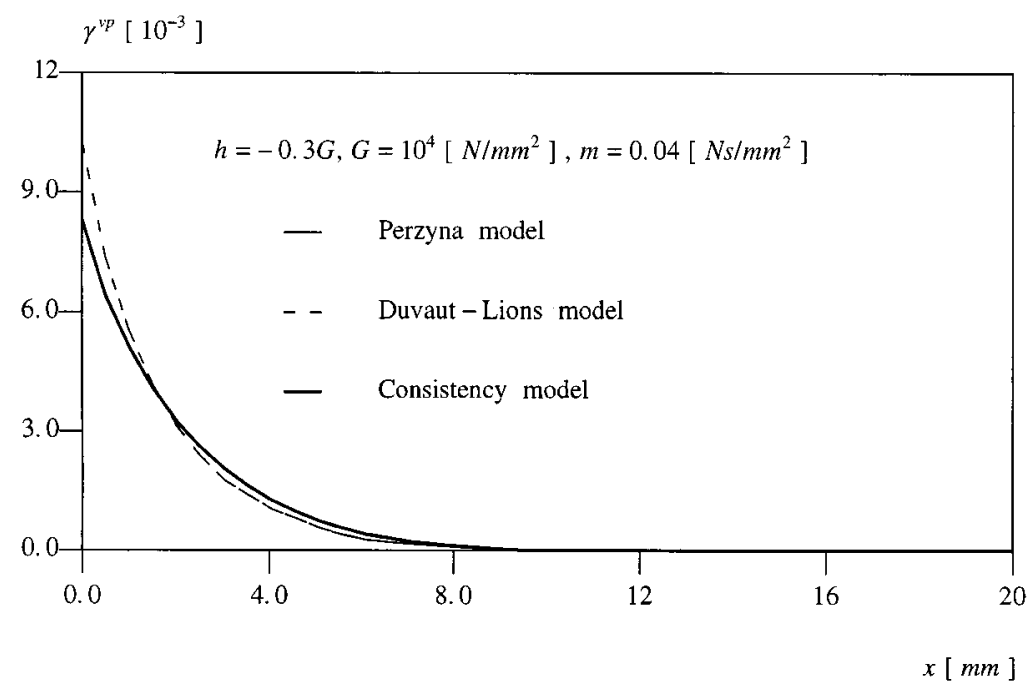

Figure 9. A comparison of viscoplastic models

Box 5. Number of global iterations of each plastic loading step for different models

\begin{tabular}{|cccc|}
\hline & Perzyna model & Duvaut-Lions model & Consistency model \\
$\Delta t / \Delta t_{\mathrm{cr}}=0.5$ & 4.0 & 4.7 & 2.0 \\
$\Delta t / \Delta t_{\mathrm{cr}}=0.2$ & 3.1 & 3.2 & 1.9
\end{tabular}

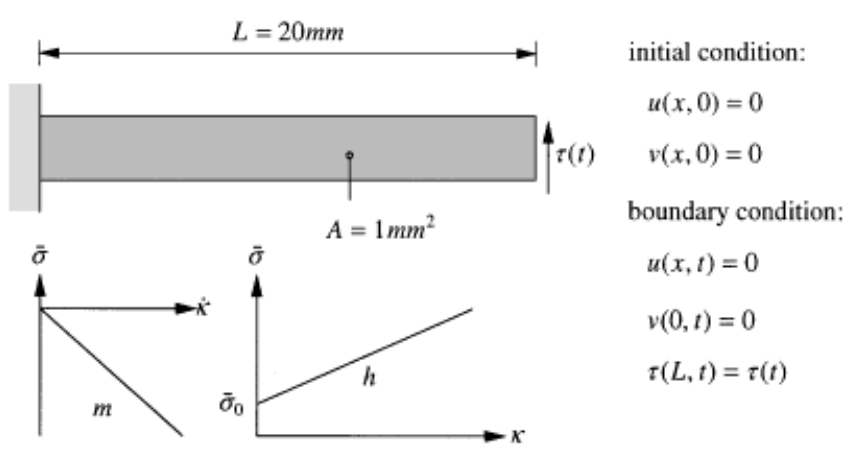

Figure 10. One-dimensional problem in shear-S-type instability

and strain-rate softening $(m<0)$ determines the propagation velocity of this shear band in the absence of temperature effects. When the deformation reaches a critical value the local PLC band is arrested due to the contribution of the strain hardening. Subsequently, a new PLC band will be initiated at the adjacent element, so that the PLC effect progresses from one end of the bar to the other in a discontinuous, but orderly and periodic fashion, Figure $11 .^{16}$ 


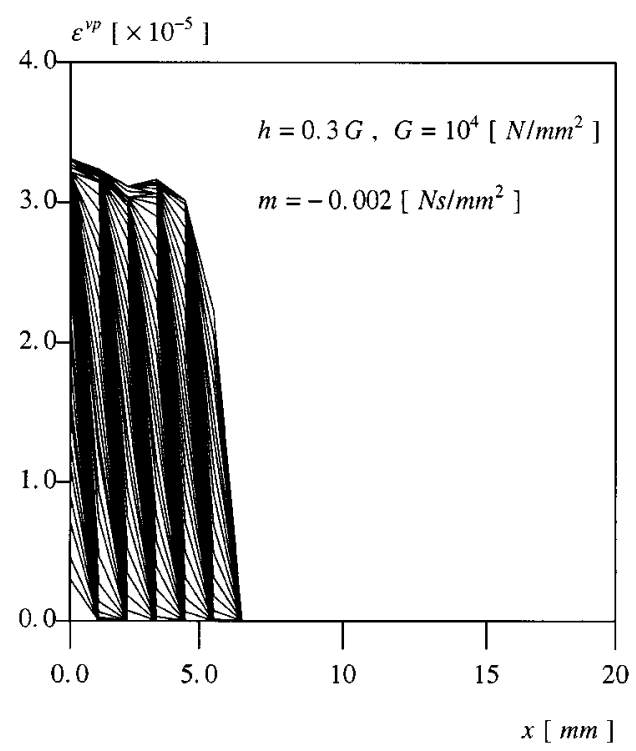

Figure 11. Propagative Portevin-Le Chatelier bands
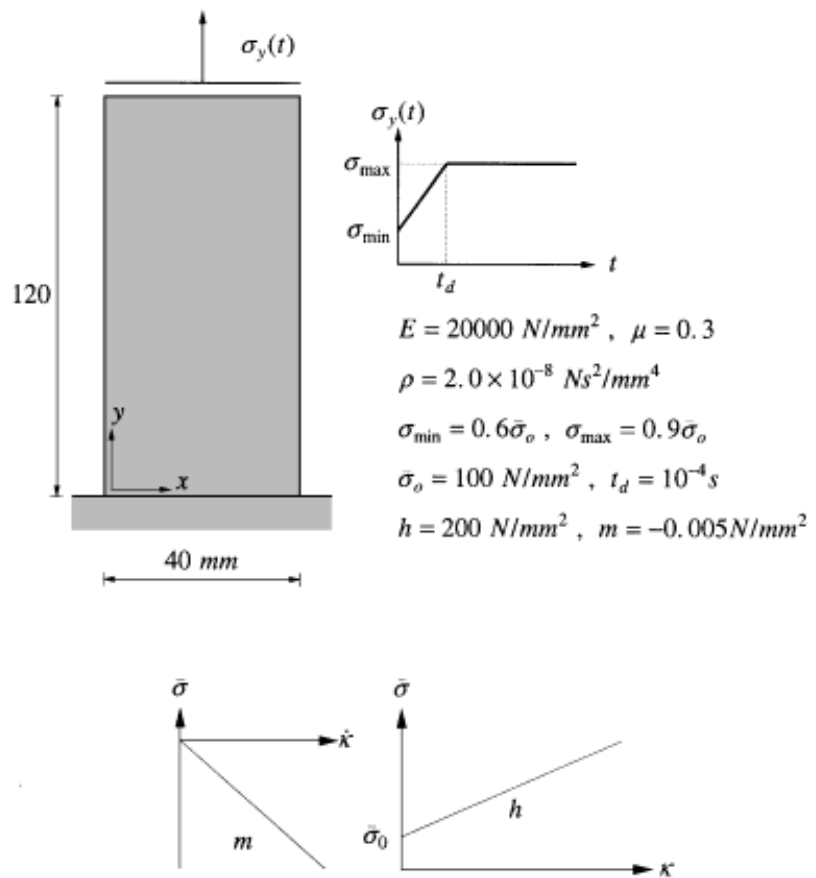

Figure 12. Biaxial test 

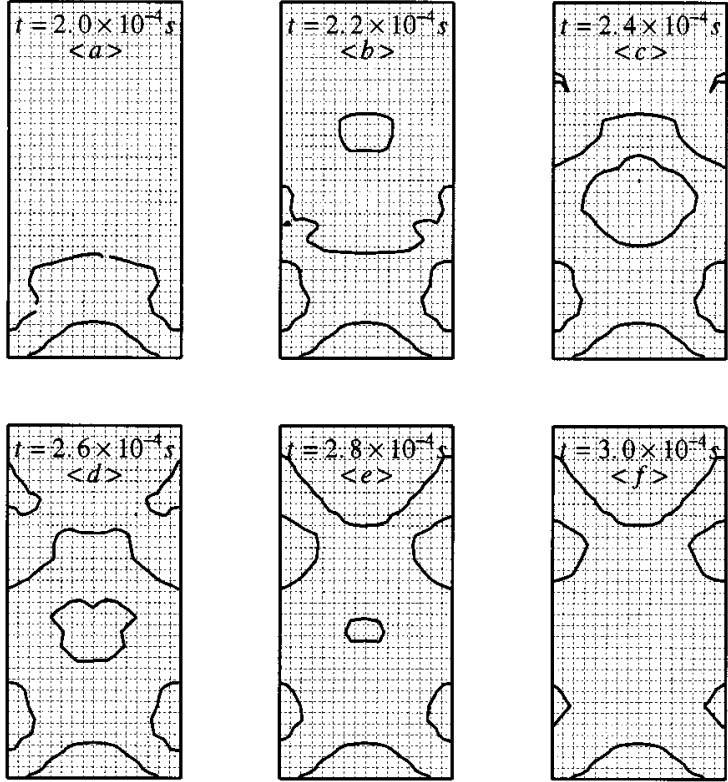

Figure 13. S-type instability: The Portevin-Le Chatelier effect. Equivalent plastic strain contours $(\kappa=0 \cdot 02)$

\subsection{Biaxial test: S-type instability}

Two-dimensional calculations of the PLC effect have been performed (Figure 12). At the top of the sample a dynamic tensile load is applied. The boundary conditions along the bottom of the sample are rigid $\left[u_{x}(x, 0)=0\right.$ and $\left.u_{y}(x, 0)=0\right]$ and the vertical displacements at the top of the sample are kept equal $\left[u_{y}(x, 120)=u_{y}(20,120)\right]$. The material parameters in Figure 12 are used in combination with a Von Mises softening plasticity model, where $\bar{\sigma}$ is the uniaxial yield stress, $\kappa$ is the equivalent plastic strain and $\mu$ is Poisson's ratio. To model the S-type instability a linear hardening model and a constant negative strain rate sensitivity is assumed. The longitudinal wave speed $c_{\mathrm{e}}=\sqrt{E / \rho}=10^{6} \mathrm{~mm} / \mathrm{s}$. For the numerical analysis we take a $16 \times 48$ mesh with plane stress elements and a nine-point Gaussian integration. The tensile loading waves will approximately propagate with an elastic wave speed $c_{\mathrm{e}}$ and the stress in the material will be doubled when reflection at the bottom takes place. Due to the boundary effect two shear bands will initiate [see Figure 13(a)] at the corner of the bottom and will develop with an inclination angle $\Theta \approx 35^{\circ}=$ (see Sluys, 1992). Due to the hardening effect further deformation leads to an increase of stress. Therefore, the first shear band remains stable and the width of the shear band enlarges when the stress waves propagate to the top, Figures 13(b) and 13(c). In this way the shear band may propagate and extend to the whole specimen, Figures 13(d)-13(f).

\section{CONCLUSIONS}

In the paper a review of rate-dependent plasticity models is given. Along with the well-known overstress laws of Perzyna and Duvaut-Lions a new model, herein named the consistency model, 
is presented in which a rate-dependent yield surface is employed. Upon viscoplastic loading the stress point remains on the yield surface in this model. For this reason the yield contour can expand/shrink not only by softening/hardening effects but also by softening rate/hardening rate effects. This model is shown to produce good results for the one- and two-dimensional simulation of propagative instabilities. These instabilities can be induced by a negative strain rate sensitivity of the material and are commonly referred to as Portevin-Le Chatelier bands. A full algorithmic treatment of the models is presented including a new algorithm for the consistency model with a derivation of a consistent tangent matrix. An algorithmic comparison between the models has been carried out. Furthermore, a relation between the length scales of the three models is derived and verified with the computational modelling of a one-dimensional shear band.

\section{APPENDIX I}

Length scale effects in viscoplastic solutions

To investigate the dispersive character of wave propagation in a viscoplastic medium, a general solution for a single linear harmonic wave with angular frequency $\omega$ and wave number $k$ is assumed

$$
v(x, t)=v_{o} \mathrm{e}^{-\alpha x} \mathrm{e}^{\mathrm{i}(k x-\omega t)}
$$

in which $v_{0}$ is a constant and $\alpha$ the spatial damping coefficient. The real non-harmonic part of the strain rate distribution is considered

$$
\dot{\gamma}=v_{0} \mathrm{e}^{-\alpha x}[-\alpha \cos (k x-\omega t)-k \sin (k x-\omega t)]
$$

because it contains the spatial attenuation part and therefore the length scale contribution. By using Taylor series expansions equation (72) gives

$$
\dot{\gamma}=-v_{0} \mathrm{e}^{-\alpha x}\left[\alpha+k(k x-\omega t)+O^{2}(k x-\omega t)\right]
$$

where $O^{n}(y)$ refers to the truncated terms of the order $y^{n}$. By considering a strain rate wave along a characteristic line $\left(x=c_{g} t\right)$ we can use

$$
k x-\omega t=\left(\frac{k}{\omega}-\frac{1}{c_{g}}\right) \omega x
$$

and equation (73) further reduces to

$$
\dot{\gamma}=-v_{0} \mathrm{e}^{-\alpha x}\left[\alpha+\frac{k}{\omega}\left(\frac{k}{\omega}-\frac{1}{c_{g}}\right) \omega^{2} x+O^{2}\left(\left(\frac{k}{\omega}-\frac{1}{c_{g}}\right) \omega x\right)\right]
$$

Substituting equation (71) into the third-order differential equation of the Perzyna model [equation (60)] and equating real and imaginary parts we can derive limit values $(\omega \rightarrow \infty)$ for the attenuation constant

$$
\alpha=\frac{3 G}{2 c_{g} m}+O^{2}\left(\frac{1}{\omega}\right)
$$


and wave number

$$
\frac{k}{\omega}=\frac{1}{c_{g}}\left[1+\frac{3 G(4 h+3 G)}{8 m^{2} \omega^{2}}\right]+O^{4}\left(\frac{1}{\omega}\right)
$$

which can be rewritten as

$$
\left(\frac{k}{\omega}-\frac{1}{c_{g}}\right) \omega x=O\left(\frac{1}{\omega}\right)
$$

If we substitute equations (76)-(78) into equation (75), we obtain

$$
\dot{\gamma}(x)=-\frac{3 v_{o} G}{2 c_{g} m} \mathrm{e}^{-3 G x / 2 c_{g} m}\left[1+\frac{4 h+3 G}{4 c_{g} m} x+O^{2}\left(\frac{1}{\omega}\right)\right]
$$

At the wave front (along the characteristic line $x=c_{g} t$ ) high angular frequencies $\omega$ dominate the solution. It seems reasonable to omit the $O^{2}(1 / \omega)$ terms in equation (79). If we define a material length scale $x=l$ as the co-ordinate at which the relative strain rate at the boundary of the shear band reads

$$
\frac{\dot{\gamma}(l)}{\dot{\gamma}(0)}=\beta
$$

where $\beta$ is a small constant representing the cut-off value of the relative strain rate at the edge of the shear band. Upon substitution of equation (80) into equation (79) the material length scale $l$ can be solved from

$$
\mathrm{e}^{-3 G l / 2 c_{g} m}\left[1+\frac{4 h+3 G}{4 c_{g} m} l\right]=\beta
$$

\section{REFERENCES}

1. Z. P. Bazant and T. B. Belytschko, 'Wave propagation in a strain softening bar-exact solution', ASCE J. Engng Mech., 111(3), 381-389 (1985).

2. A. Needleman, 'Material rate dependence and mesh sensitivity on localisation problems', Comput. Methods. Appl. Mech. Engng., 67, 69-86 (1988).

3. B. Loret and J. H. Prevost, 'Dynamic strain localisation in elasto-(visco-) plastic solids, Part 1. General formulation and one-dimensional examples', Comput. Methods Appl. Mech. Engng., 83, 247-273 (1990).

4. L. J. Sluys, 'Wave propagation, localisation and dispersion in softening solids', Doctoral Thesis, Delft University of Technology, The Netherlands, 1992.

5. W. M. Wang, L. J. Sluys and R. de Borst, 'Interaction between material length scale and imperfection size for localisation in viscoplastic media', Eur. J. Mech., A/Solids, 15(3), 447-464 (1996).

6. T. B. Belytschko, X. Wang, Z. P. Bazant and Y. Hyun, 'Transient solutions for one-dimensional problems with strain softening, J. Appl. Mech., 54, 513-518 (1987).

7. P. Perzyna, 'Fundamental problems in viscoplasticity', in Recent Advances in Applied Mechanics, vol. 9, Academic Press, New York, 1996, pp. 243-377.

8. I. C. Cormeau, 'Numerical stability in quasi-static elasto/visco-plasticity', Int. J. Numer. Meth. Engng., 9, 109-127 (1975).

9. D. R. J. Owen and F. Damjanic, 'Viscoplastic analysis of solids: stability consideration', in Recent Advances in Non-linear Computational Mechanics, Pineridge Press Ltd., Swansea, 1982, pp. 225-253.

10. G. Duvaut and J. L Lions, Les Inequations en Mechanique et en Physique, Dunod, Paris, 1972.

(C) 1997 John Wiley \& Sons, Ltd.

Int. J. Numer. Meth. Engng., 40, 3839-3864 (1997) 
11. J. C. Simo, J. G. Kennedy and S. Govindjee, 'Non-smooth multisurface plasticity and visco-plasticity-Loading/unloading conditions and numerical algorithms', Int. J. Numer. Meth. Engng., 26, 2161-2185 (1988).

12. Y. Estrin, L. P. Kubin and E. C. Aifantis, 'Introductory remarks to the viewpoint set on propagative plastic instabilities', Scr. Metall., 29, 1147-1150 (1993).

13. T. J. R. Hughes, The Finite Element Method-Linear Static and Dynamic Finite Element Analysis, Prentice-Hall, Englewood Cliffs, N.J., 1987.

14. A. H. van den Boogaard, 'Implicit integration of the Perzyna viscoplastic material model', TNO Report, 95-NM-R711, The Netherlands, 1995.

15. J. W. Ju, 'Consistent tangent moduli for a classic of viscoplasticity' ASCE J. Engng. Mech., 116(8), 1764-1779 (1990).

16. W. M. Wang, L. J. Sluys and R. de Borst, 'Computational modelling of dynamic instabilities in metals', in D. R. J. Owen et al. (eds.), Proc. COMPLAS IV, Barcelona, 1995, pp. 1877-1888. 